\title{
A Experiência de Implantação de uma Disciplina Maker em uma Escola de Educação Básica
}

\author{
Eduardo Borges Gomes ${ }^{1}$, André Luís Alice Raabe ${ }^{123}$, André Luiz. Maciel Santana ${ }^{1}$, \\ Raphael da Silva ${ }^{1}$, Julia Peron Metzger ${ }^{1}$, Marli Fátima Vick Vieira ${ }^{24}$ \\ ${ }^{1}$ Laboratório de Inovação Tecnológica na Educação - CTTMar - UNIVALI \\ ${ }^{2}$ Programa de Pós-Graduação em Educação \\ ${ }^{3}$ Mestrado em Computação Aplicada \\ ${ }^{4}$ Instituto Federal de Educação, Ciência, e Tecnologia Catarinense - (IFC) \\ raabe@univali.br, andrelms@univali.br, litedunivali.br,
marli.vieiradifc.edu.br
}

\begin{abstract}
The paper reports the implementation of a Maker discipline in a School of Application linked to a Brazilian University. The proposal of Maker activities (hands on) has attracted the attention of educators by providing an environment where it is possible to interweave concept learning with practices that stimulate the protagonism of students. The article seeks to detail the school environment, the conception of the discipline, the organization of the space and the pedagogical planning so that it can contribute to other institutions that wish to adopt practices Maker in their curricula can have a reference in progress considering the educational, social and Economic development of our country.
\end{abstract}

Resumo. O artigo relata a implantação de uma disciplina Maker em um Colégio de Aplicação vinculado a uma Universidade brasileira. A proposta de atividades Maker (mão na massa) tem atraído atenção de educadores por proporcionar um ambiente onde é possível entrelaçar a aprendizagem de conceitos com práticas que estimulam o protagonismo dos estudantes. $O$ artigo busca detalhar o ambiente escolar, a concepção da disciplina, a organização do espaço e o planejamento pedagógico de forma que possa contribuir para outras instituições que desejem adotar práticas Maker em seus currículos e possam ter uma referência em andamento considerando a realidade educacional, social e econômica do nosso país.

\section{Introdução}

O Movimento Maker é uma extensão da cultura DIY (Do-It-Yourself/Faça-Você-Mesmo) e tem em sua base a ideia de que pessoas comuns podem construir, consertar, modificar e fabricar os mais diversos tipos de objetos e projetos. A Adoção de atividades Maker (ou mão na massa) na Educação tem se tornado 
uma tendência em diferentes países e também no Brasil. Multiplicam-se projetos experimentais para levar atividades de curta ou média duração para escolas. Redes de pesquisadores e entusiastas se organizam em torno do tema como a Rede de Aprendizagem Mão na Massa ${ }^{1}$ e a rede em torno do evento FabLearn ${ }^{2}$, que ocorre desde 2011 no EUA e passou a ser realizado também no Brasil em 2016.

Conforme Dougherty (2012), o avanço e a disponibilidade das novas tecnologias de fabricação digital estão entre os fatores que mais tem impulsionado o crescimento do movimento. A redução do custo de equipamentos como Impressoras 3D, kits robóticos, Fresadoras CNC e Cortadoras Laser tem permitido que estes equipamentos sejam utilizados em atividades de propósito educacional, criando novas configurações de exploração do uso de tecnologia e de informática na Educação.

Do ponto de vista pedagógico, a maioria da atividades maker se fundamenta na abordagem Construcionista (Papert, 1980), que enaltece os benefícios do envolvimento do estudante em projetos em que ele assume o protagonismo e promove a criação de algum objeto que possa ser socializado. As decorrências desta abordagem são profundas na organização de atividades educacionais. Como salienta Dougherty (2012 p.5) "nós deveríamos parar de perguntar como podemos testar o conhecimento do estudante e passar a perguntar o que o estudante pode fazer com o que ele sabe. Quando ele constrói um artefato, este objeto é a demonstração do que ele aprendeu, o objeto provê a evidência da aprendizagem".

Portanto, levar a cultura maker de forma sistemática e perene para educação básica significa enfrentar desafios de ordem econômica, curricular, pedagógica e organizacional. Poucas são as referências sobre como adotar atividades maker em escolas no Brasil e as dúvidas sobre o investimento, espaço, faixa etária atendida, riscos envolvidos, planejamento pedagógico, avaliação e relação com o currículo estão apenas começando a ser respondidas.

Nesta direção o presente artigo contribui ao apresentar a experiência de implantação de um disciplina maker no currículo de um Colégio de Aplicação da Universidade do Vale do Itajaí (CAU) enfatizando as decisões referentes a concepção da disciplina, a organização do espaço, proposta pedagógica e os primeiros resultados provenientes da participação dos estudantes de fevereiro a junho de 2017.

O artigo está organizado em seções, a seção 2 descreve o ambiente escolar onde a disciplina foi implantada. A seção 3 descreve a preparação e organização do espaço e equipamentos. A Seção 4 descreve o planejamento pedagógico e a seção 5 apresenta os resultados e impactos da iniciativa.

\section{O Ambiente Escolar e a Criação de uma Disciplina Maker}

O CAU (Colégio de Aplicação da Universidade do Vale do Itajaí) é um ambiente

1 http://redemaonamassa.org/

2 http://fablearn.org/conferences/ 
educacional de articulação entre o ensino básico e o superior, que incentiva o ensino, a pesquisa e a extensão, propiciando a formação de profissionais e o desenvolvimento de materiais didáticos-pedagógicos. Esse espaço possui estudantes desde a educação infantil até o ensino médio e possibilita a eles o contato com toda a estrutura de uma universidade, fazendo com que tenham uma visão do que é estar em um contexto universitário.

Antecipando-se às mudanças da novas políticas públicas do Brasil, em especial a reformulação do Ensino Médio, o CAU reorganizou sua matriz curricular. O foco desta reestruturação foi para que, no ano de 2017, fosse adotada a modalidade de ensino integral a partir do sexto ano do ensino fundamental (Fundamental II) até o terceiro ano do ensino médio. Com isso, foram incluídas disciplinas obrigatórias e optativas sendo que, das optativas, caberia aos estudantes a escolha de ao menos 2 (duas), para que fossem cursadas no período oposto às suas atividades regulares.

Neste movimento de adaptação à nova legislação, pesquisadores de Informática na Educação da Universidade assessoraram a equipe pedagógica do Colégio recomendando que fossem incluídas duas disciplina. A primeira, denominada Laboratório Maker, é voltada a proporcionar a vivência de projetos "mão na massa", que combinam diferentes modalidades construtivas, como marcenaria, costura, robótica, impressão em 3D, entre outros; já a segunda, denominada Pensamento Computacional, é voltada ao desenvolvimento da fluência no uso de conhecimentos de Computação para resolução de problemas, bem como a criação de aplicações por meio da programação. A proposta do presente artigo, no entanto, é explorar a experiência referente a disciplina Laboratório Maker, como será visto na sequência.

As recomendações foram bem recebidas pela equipe pedagógica do colégio e passaram a ser desenhadas de forma conjunta com os pesquisadores do Grupo de Informática na Educação e do Laboratório de Inovação Tecnológica na Educação - LITE da Universidade. Ainda que as crenças pedagógicas dos pesquisadores fossem de que o formato de disciplina não era o mais adequado para realização de atividades maker, foi o formato possível de ser implantado tendo em vista a estrutura organizacional do colégio.

A disciplina foi definida tendo dois períodos consecutivos (de 50 minutos cada) totalizando 1h:40m (uma hora e quarenta minutos), sendo oferecida uma vez por semana para cada turma. As turmas possuem no máximo 18 estudantes e são organizadas mesclando estudantes de diferentes anos e idades dentro de um mesmo nível (fundamental II ou ensino médio). A oferta foi realizada para todos os estudantes do sexto ano do ensino fundamental até o terceiro ano do ensino médio. Ficou definido ainda que o espaço de realização das atividades seria o LITE que portanto passaria por uma adaptação para receber os estudantes.

\section{A Organização do Espaço Maker}

Para atender ao colégio foi decidido realizar uma ampliação no espaço do LITE. Esta decisão levou em conta que o espaço já estava parcialmente ajustado a cultura maker, 
VI Congresso Brasileiro de Informática na Educação (CBIE 2017)

Anais do XXIII Workshop de Informática na Escola (WIE 2017)

possuindo os equipamentos de custo mais alto (impressora 3D e cortadora laser) adquiridos por meio de verba de projetos de pesquisa e extensão.

Outro aspecto particular do laboratório é a atuação de professores de graduação e pós-graduação, alunos da graduação dos cursos da Ciência da Computação, Engenharia da Computação, Mecânica, Produção, Design e Arquitetura, como também pós-graduandos em nível de mestrado e doutorado, das áreas da Ciência da Computação e Educação. De forma que viabilizar o convívio e a participação eventual destes nas atividades realizadas com os estudantes do colégio tornou-se uma diretriz para a reforma do espaço.

A Figura 1 ilustra a planta do espaço que é bipartido, a parte direita corresponde ao espaço maker onde as atividades da disciplina são realizadas, e o espaço a esquerda corresponde ao ambiente de convívio dos pesquisadores e estudantes de graduação.

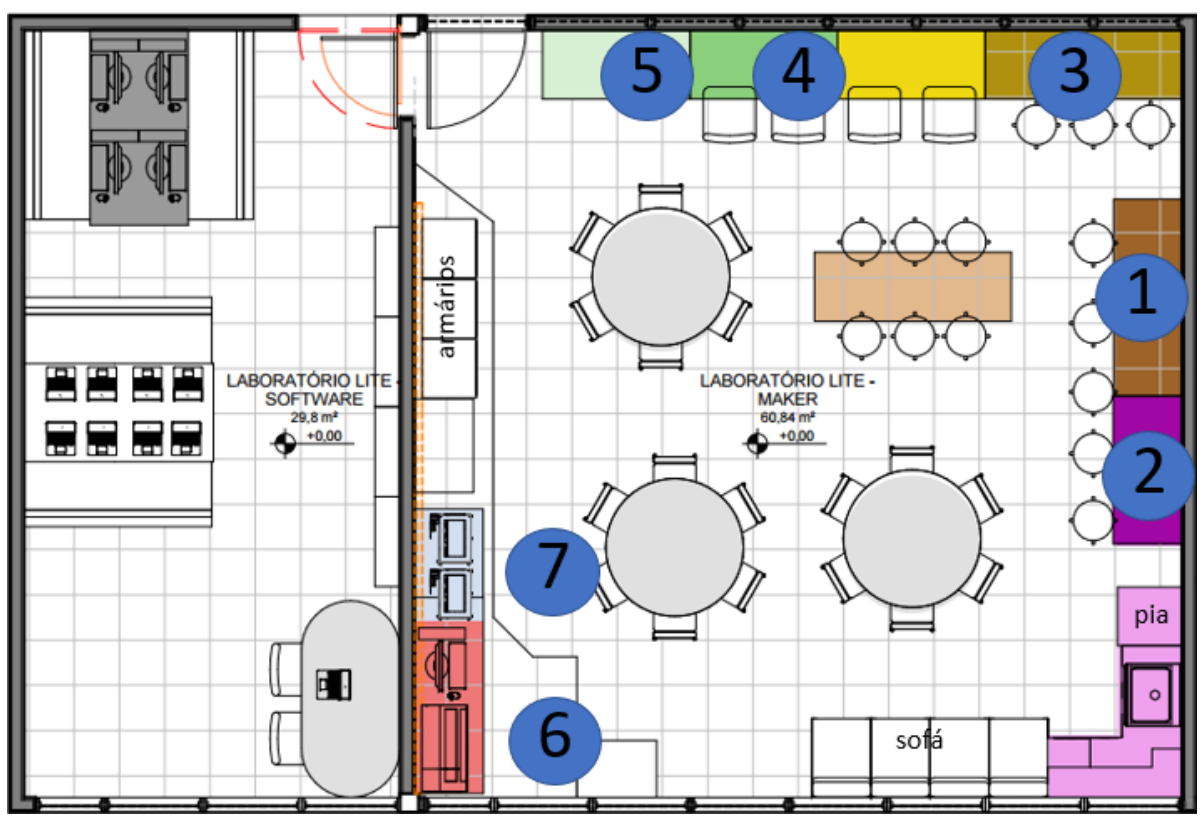

Figura 1 - Planta Baixa do Laboratório

O espaço foi organizado baseado na experiência relatada em Blikstein (2013) e Santana et al. (2016) e usando o conceito de estações como sendo um espaço que concentra os equipamento e materiais para o trabalho com determinada tecnologia inspirado em Doorley (2016). Na planta é possível observar as sete estações numeradas sendo elas: (1) Artesanato em papel (papercraft), (2) Costura, (3) Eletrônica, (4) Marcenaria, (5) Registros, (6) Corte a Laser e (7) Impressão 3D. Além das estações o laboratório conta com uma pia e um sofá. A área do espaço maker compreende $60 \mathrm{~m}^{2}$. O local foi organizado tendo como referência espaços visitados pelos pesquisadores do LITE, em especial no Media Lab do Massachusetts Institute of Technology (MIT) ${ }^{3}$ e na Universidade de Stanford ${ }^{4}$.

3 Maiores informações disponíveis em <web.mit.edu>.

4 Maiores informações disponíveis em <www.stanford.edu>. 
Assim, o espaço prioriza mesas adequadas ao trabalho em grupo e que comportem o uso de computadores portáteis. Foram instaladas tomadas acessíveis nas mesas para que cada mesa possa comportar 6 estudantes usando dois notebooks (um notebook a cada 3 estudantes). $\mathrm{O}$ ambiente não tem um quadro para explicações expositivas, para este fim utiliza-se uma TV plana de 40 polegadas sobre um suporte móvel que se desloca pelo laboratório aproximando-se das mesas quando necessário.

Quanto ao custo de montagem do ambiente, este não é possível de ser auferido com exatidão, tendo em vista o reaproveitamento de equipamentos e móveis que já existiam no laboratório. O quadro 1 apresenta os principais equipamentos que constam no espaço. Estima-se que a para montagem de um ambiente com os mesmos equipamentos em uma sala desprovida de qualquer móvel seja em torno de 50 mil reais (valor estimado em junho de 2017).

Quadro 1 - Equipamentos do Espaço Maker

\begin{tabular}{|l|c|l|c|}
\hline Equipamento & Qtd & Equipamento & Qtd \\
\hline Impressora 3d & 2 & Plotter de Corte pequena & 1 \\
\hline Cortadora Laser & 1 & Máquina de Costura & 1 \\
\hline Notebooks & 6 & Kits Robóticos & 10 \\
\hline Filmadora & 1 & Micro Retífica & 1 \\
\hline Lixadeira & 1 & Furadeira & 1 \\
\hline Ferramentas de marcenaria & 8 & Televisor 40' & 1 \\
\hline
\end{tabular}

\section{O Planejamento Pedagógico}

O LITE conduz desde o início de 2015 um projeto de extensão em parceria com uma escola pública municipal. O nome deste projeto foi eleito pelos seus primeiros participantes como Lite Is $\mathrm{Cool}{ }^{5}$. No ano de 2017 está sendo realizada a terceira edição, passando nesse período por mudanças significativas. Esta experiência prévia auxiliou a definir as decisões pedagógicas para atender a disciplina Laboratório Maker.

Estabelecido os parâmetros iniciais da disciplina, foram elaboradas estratégias com o intuito de fazer com que os estudantes, de forma independente pudessem ter acesso a todas as estações contidas no laboratório para a realização de atividades maker.

Ao final de discussões feitas por alguns integrantes do Laboratório Maker a ementa da disciplina teve como objetivo geral possibilitar a estudantes concretizar projetos que envolvem a concepção e construção de artefatos combinando diferentes tecnologias construtivas, materiais e técnicas.

Foi pensado em atividades que possam ser realizadas no espaço dedicado às atividades e estimulam a autonomia, colaboração e criatividade dos participantes, para isso, criou-se um método constituído por desafios. Cada desafio envolve ao menos uma das áreas preestabelecidas, que são: costura, marcenaria, programação, eletrônica, papercraft, audiovisual e modelagem. Tais desafios instigam o uso de ferramentas e máquinas existentes no laboratório, disponíveis de forma separada nas estações. Ainda,

\footnotetext{
${ }^{5}$ Veja mais informações sobre o projeto no artigo de Santana et al.(2016).
} 
VI Congresso Brasileiro de Informática na Educação (CBIE 2017)

Anais do XXIII Workshop de Informática na Escola (WIE 2017)

cabe aos estudantes a escolha de realizá-los em grupo ou de forma individual, sendo que alguns devem ser, obrigatoriamente, individuais.

As áreas abordadas na disciplina foram escolhidas pelos próprios realizadores, podendo ser modificadas de acordo com a singularidade de cada espaço onde a disciplina será realizada.

Divididos em níveis de dificuldade, alguns dos desafios exigem a conclusão de outros, chamados de pré-requisitos, para serem finalizados. Tal sistemática facilita a criação de uma linha de conhecimento de forma gradativa por parte do aprendiz.

Tem-se, então, como exemplo de desafio com pré-requisito, aquele que aborda o uso da máquina de costura, em que o estudante deve realizar, ao menos, um ponto de costura de forma manual.

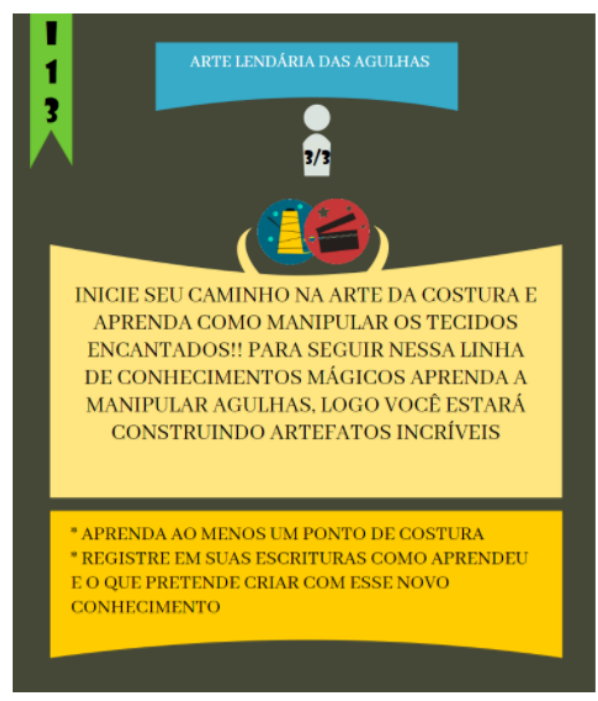

Figura 2 - Desafio com Pré-requisito.

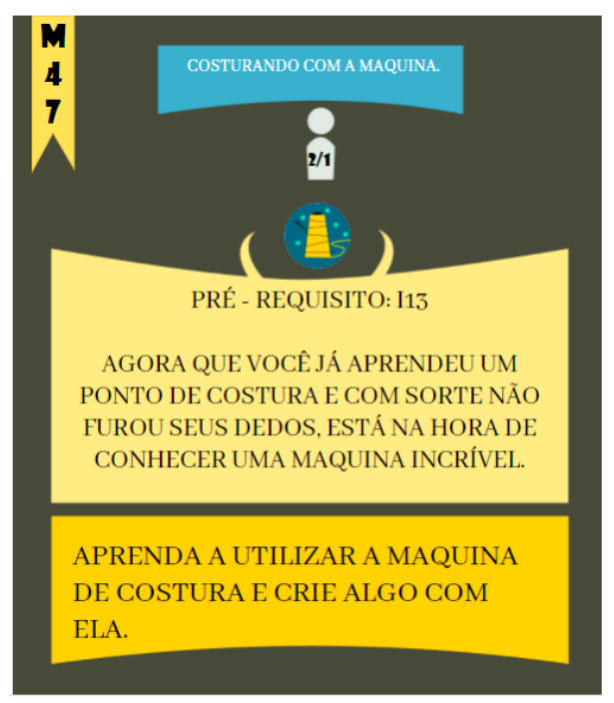

Figura 3 - Desafio sem Pré-requisito.

Ao realizar um desafio, ou até mesmo no andamento do mesmo, o aprendiz deve registrar suas atividades em um blog pessoal. Podendo conter fotos, vídeos, ou qualquer tipo de mídia que o blog suportar, realizar o registro é requisito obrigatório para que o estudante complete o desafio.

Não existe uma regra estipulada que impeça aprendizes abortarem desafios iniciados por eles, a qualquer momento um estudante pode começar um novo desafio.

A questão de como seriam avaliados os estudantes por meio das atividades realizadas na disciplina foi um tópico discutido durante a concepção do modelo, considerando que não existe uma linha de conhecimento predefinida que o aprendiz terá que seguir durante o ano letivo, foi difícil criar um padrão de avaliação.

A obrigatoriedade de uma avaliação por meio de notas é decorrente da disciplina ser curricular em um colégio que tem como padrão avaliativo este método, porém foram feitos ajustes necessários para que isso não tornasse as atividades uma obrigação do aprendiz, indo contra características da aprendizagem construcionistas Na perspectiva 
da proposta construcionista, avaliar é acompanhar e valorizar todo o processo de construção do conhecimento vivenciado pelo estudante, sendo que esta avaliação é feita qualitativamente. Decidiu-se então usar os registros das atividades, feitos pelos próprios aprendizes como ferramenta de avaliação individual, em que seriam observados aspectos como: qualidade dos detalhes registrados e conhecimento demonstrado sobre o assunto abordado na atividade realizada.

Os desafios abordam temas e áreas diferentes para faixas-etárias distintas, uma turma com estudantes de 16 a 18 anos de idade recebem desafios diferentes de uma turma com estudantes de 13 a 15 anos, por exemplo.

Vários desafios foram disponibilizados no início das atividades da disciplina e ao decorrer do período letivo, são adicionados novos, de acordo com o andamento de cada turma. Dessa forma, o aprendiz pode escolher qual área de conhecimento quer abordar sem ter que seguir uma linha de atividades pré definida. Isso faz com que estudantes de uma mesma disciplina, em um mesmo espaço, aprendam diferentes conteúdos e com diferentes estratégias.

Semanalmente é conduzida entre os três facilitadores responsáveis pela condução das turmas uma reunião para discutir as ocorrências existentes na semana, replanejar atividades e disponibilizar novos desafios. Essa reunião é aberta para qualquer integrante do LITE e acontece no próprio laboratório.

Vale citar que para a criação dos desafios é utilizado uma página web criada por um dos integrantes do laboratório. Nesse ambiente são inseridos textos que depois são adaptados para serem disponibilizados nos modelos de desafios.

\subsection{Aplicação das Atividades}

No início das atividades com os estudantes, foi apresentada a disciplina, juntamente com o método de aprendizagem, também foi entregue aos aprendizes cadernos contendo regras de manuseio dos equipamentos disponíveis no laboratório e uma tabela dividida pelas áreas dos desafios. Nos primeiros encontros, os aprendizes ainda estavam com receios em relação a disciplina, muitos deles esperavam instruções dos facilitadores para a realização dos desafios. Após o momento de adaptação, a escolha e realização das atividades tornou-se algo natural para os estudantes.

Ao participar das atividades os estudantes se dividem em grupos, reúnem-se ao redor das mesas e verificam o grupo de desafios que estão disponibilizados online, por meio dos seus smartphones ou notebooks disponíveis no laboratório. Ao escolher qual desafio deseja realizar no dia, direcionam-se a estação da área que atende suas necessidades e começa a executá-las. $\mathrm{O}$ aprendiz também pode continuar o desafio que deixou em andamento na aula passada, assim que concluí-lo seleciona um novo. Ao completar um desafio, o estudante carimba seu caderno e dependendo do que foi criado pode levar o produto final gerado por ele para casa.

Durante o primeiro semestre da disciplina os aprendizes desenvolveram diversos 
VI Congresso Brasileiro de Informática na Educação (CBIE 2017)

Anais do XXIII Workshop de Informática na Escola (WIE 2017)

projetos, fazendo uso das estações do laboratório, entre os projetos criados foi possível observar estudantes que até então não possuíam conhecimento sobre eletrônica ou programação criarem aplicações envolvendo o Arduino. Outros que nunca tiveram contato com costura criaram almofadas em conjunto com outros participantes que até então não possuíam afinidade entre sí, mesmo pertencendo a mesma turma. Esse tipo de ocorrência aconteceu com os mais diversos grupos de estudantes, que ao concluir desafios se depararam com conhecimentos que não obtinham até então.

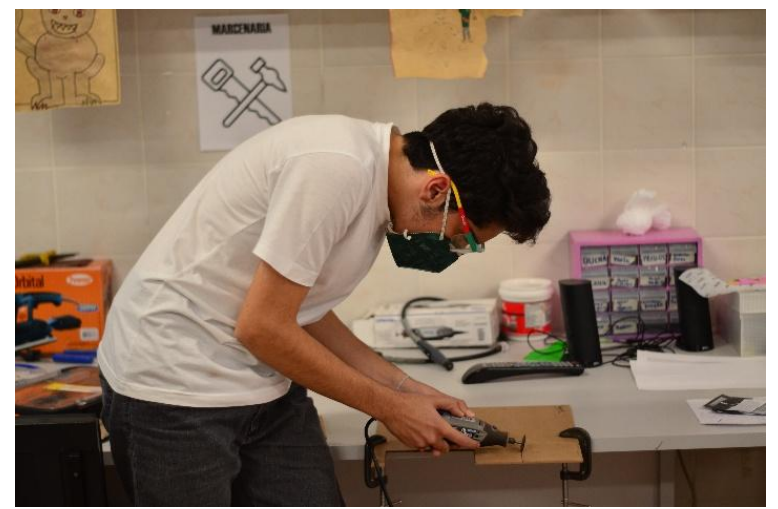

Figura 4- Aprendiz Utilizando Estação de Marcenaria.

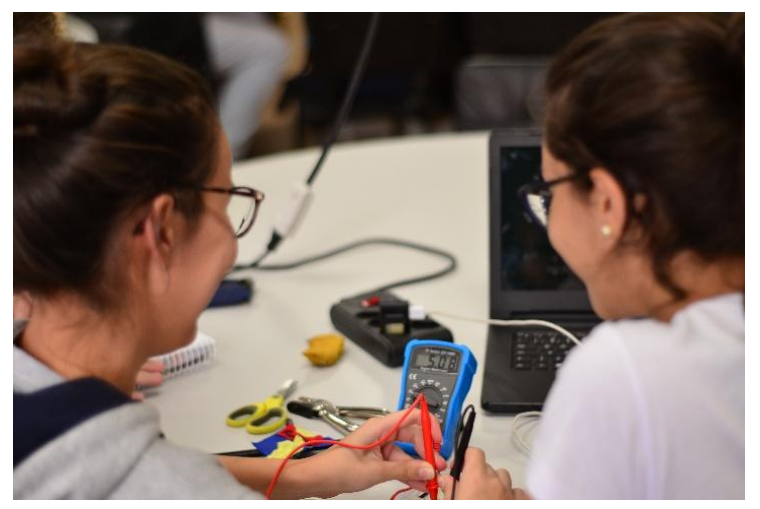

Figura 5- Aprendizes Trabalhando Colaborativamente.

Após a conclusão de um desafio os aprendizes registram em um blog individual, (https://www.tumblr.com/), compartilhando a experiência vivenciada. Nesse espaço facilitadores e demais integrantes podem verificar relatos dos próprios estudantes sobre atividades realizadas e experiências obtidas.

No espaço onde são feitos os registros é possível verificar o número de publicações feitas por cada estudante, em média é realizado no mínimo um registro por aula por cada aprendiz.

Sendo possível um aprendiz seguir os blogs dos outros integrantes, o compartilhamento de registros gera um interesse maior em mostrar aquilo que é feito. 


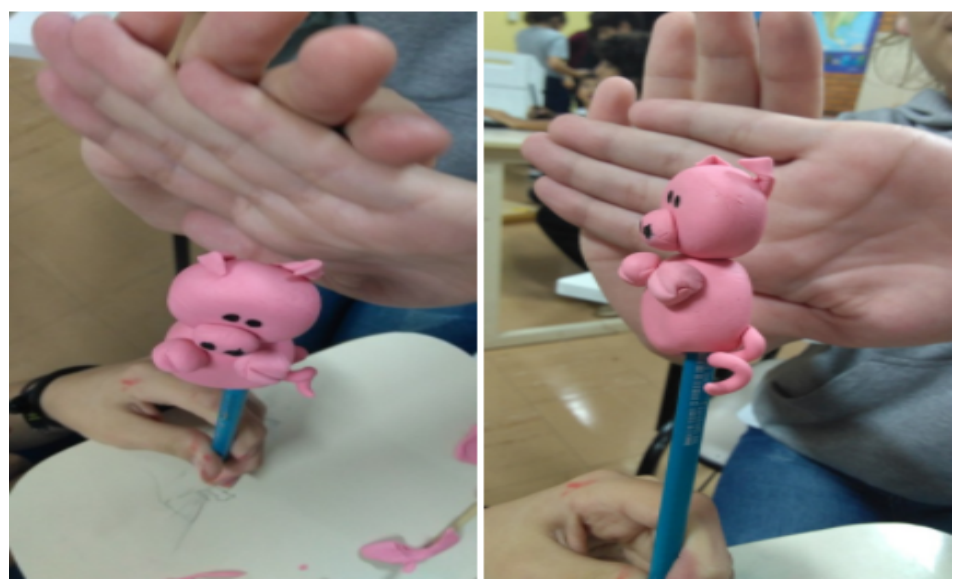

Figura 6- Registro Feito por um Aprendiz em seu Blog Pessoal.

Os facilitadores não interferem diretamente nas atividades que são realizadas pelos estudantes. Dessa forma, podem reunir esforços para compreender a evolução de cada turma, que depois serão discutidas nas reuniões semanais, com o objetivo de elaborar novos desafios.

\section{Conclusões}

Apesar das dificuldades iniciais por conta das mudanças curriculares ocorridas no colégio obtivemos um número de significativo de de alunos inscritos na disciplina Laboratório Maker, essa ocorrência indica que interesse por atividades makers está presente entre os jovens.

A possibilidade de realizar uma atividade que seja de interesse pessoal do aprendiz, entre as diversas áreas, sem estipular uma linha obrigatória a ser seguida faz com que um grupo de aprendizes consigam aprender conhecimentos diferentes uns dos outros e de formas diferentes, em um espaço e ao mesmo tempo. Essa particularidade é possível devido a diversidade de áreas envolvidas por meio de seus integrantes e da concepção de um ambiente semelhante ao LITE.

Tornar obrigatório os registros dos estudantes, além de auxiliar os facilitadores a entenderem como estão realizando as atividades, faz com que os aprendizes tenham consciência do que está sendo realizado e do caminho de conhecimento que estão construindo.

As atividades propostas nessa disciplina, exigem a preparação de um ambiente que possibilite alocação de recursos de diferentes áreas, para que no momento de realização das atividades a procura pelo equipamento ou matéria prima necessária seja de fácil acesso.

As experiências obtidas com o Lite Is Cool permitiram a evolução de ideias sobre a aplicação de atividades makers e desta forma possibilitou o aprimoramento das mesmas e a implementação da disciplina Laboratório Maker.

As tecnologias e ferramentas existentes no laboratório impossibilita o número elevado de aprendizes ao mesmo tempo, o colégio entendeu tal particularidade e 
VI Congresso Brasileiro de Informática na Educação (CBIE 2017)

Anais do XXIII Workshop de Informática na Escola (WIE 2017)

possibilitou que as turmas fossem divididas em grupos menores do que as disciplinas existentes no currículo anterior ao de 2017.

Os facilitadores passaram a atuar como coadjuvantes no processo de aprendizagem dos estudantes nas atividades propostas, o foco é a atuação dos aprendizes como protagonistas. O papel do facilitador é anterior às atividades realizadas no laboratório, planejando e elaborando os desafios, sendo facilitador do processo do conhecimento no decorrer das atividades.

Espera-se que atividades como essa possam servir de inspiração sobre como proporcionar o desenvolvimento do pensamento computacional em atividades maker que seguem pressupostos construcionistas.

\section{Referências}

Blikstein, P. (2013). Digital Fabrication and 'Making' in Education: The Democratization of Invention. In J. Walter-Herrmann \& C. Büching (Eds.), FabLabs: Of Machines, Makers and Inventors. Bielefeld: Transcript Publishers.

Dougherty, Dale. (2012) The Maker Movement. Innovations: Technology, Governance, Globalization V.7, N.3,

Doorley, Scott et al. (2011). "Make space:" How to set the stage for creative collaboration. John Wiley \& Sons..

Papert, S. (1980). Mindstorms : children, computers, and powerful ideas. New York: Basic Books.

Santana, André., Raabe, André., Santana, Luís F., Metzger, Júlia, Gomes, Eduardo B., Vieira, M. F. V. (2016) Atividades Maker no Processo de Criação de Projetos por Estudantes do Ensino Básico para uma Feira de Ciências. In: Anais do XXII Workshop de Informática na Escola (WIE 2016). Uberlândia: SBC, 2016.

Santana, André. L. m. ; Raabe, A. ; Santana, L. F.; Ramos, G.; Vieira, M. V.; Santos, A. A. (2016) Lite Maker: Um Fab Lab móvel para aplicação de atividades mão na massa com estudantes do ensino básico. In: Anais do XXII Workshop de Informática na Escola (WIE 2016). Uberlândia: SBC, 2016. 